\title{
How modern data management software and state of the art inspection gauges improve the efficiency of the pipeline coating inspection process
}

\author{
David Barnes* \\ Elcometer Ltd, Manchester M43 6BU, UK
}

\begin{abstract}
The inspection of pipeline coating is crucial to the lifetime performance of the pipeline. Inspection during installation of the pipeline and as part of the routine maintenance programme is essential. It is often said that inspection processes save money by ensuring that relevant specifications are achieved but that writing reports for the inspection process cost money. One way to reduce the cost of inspection reporting and to speed up the inspection process is to use a data management system to present the inspection data in a consistent and organised manner. The automation of the reporting process is an important cost saving that allows more time to be allocated to the important task of inspection and the achievement of the coating specification. There have been recent developments in both the design of reporting software and inspection gauges which together make achieving a paperless quality assurance system a reality for all protective coating applications. This paper describes the latest design and operational features of coating thickness gauges, dewpoint meters, surface profile gauges and other related gauges and describes how data can be easily transferred from the memory of these gauges into personal computers and mobile devices by running a dedicated software program for coating inspection data management. The creation of reports combining test results from a broad range of both digital and non-digital test methods will be discussed with particular emphasis on the use of Standard reports and the preparation of preformatted

report

forms.
\end{abstract}

\section{Introduction}

During the coating of steel structures several inspection measurements are required to be taken and reported in accordance with whichever standard the installer is required to follow.

ISO 19840 Paints and varnishes -- Corrosion protection of steel structures by protective paint systems -- Measurement of, and acceptance criteria for, the thickness of dry films on rough surfaces and ISO 8502-4 Preparation of steel substrates before application of paints and related products -- Tests for the assessment of surface cleanliness -- Part 4: Guidance on the estimation of the probability of condensation prior to paint application are but two of the standards which may be used.

As important in this process is the reporting of the findings. Gauges are now designed with memory and connectivity capability to simplify and accelerate this procedure.

\section{Coating Inspection}

The process of applying coatings to steel structures is monitored from the preparation of the substrate, through the climatic conditions prior to application, the applied coating thickness and various properties of the dry film.

Before coating the surface needs to be prepared such that the required profile is applied and the cleanliness of the surface is confirmed to comply with the specification requirements of the coating.
The blast profile can be measured in various ways but one of the simplest and fastest ways to measure profile is to use a digital profile gauge.

The gauge is placed on the surface and the spring mounted needle measures the depth of the valley in which it lands relative to the peaks on to which the gauge is placed. Obviously measuring one valley on a blasted surface will not characterise the surface, so several measurements are taken (10 according to ASTM D 4417).

In order to characterise the whole surface under inspection correctly, the average of these 10 readings should be taken to give a more accurate assessment. This aligns with good Statistical Analysis practice.

SSPC PA 17 states that "Unless otherwise specified, select a minimum of three $15 \times 15 \mathrm{~cm}$ (or three $6 \times 6$ inch) locations in which to take readings for each specific surface preparation apparatus used during each work shift or twelve-hour period, whichever is shorter."

In more recent times these gauges have been digitised and whilst they work on the same principle, data management and statistical analysis within the gauge is now possible. These gauges are now capable of having the measuring or contact point at the end of a cable to enable access in more hard to reach locations. The measurement procedure remains the same.

Having achieved the desired profile, the cleanliness of the substrate needs to be assessed.

A new design of conductivity meter based on microprocessor electronic circuitry has been developed

${ }^{*}$ Corresponding author: david.barnes $@$,elcometer.com 
with features to make the assessment of surface contamination by soluble salts quick and easy.

\section{The saturated filter paper extraction method}

Soluble salt measurements in general require two processes, the first is to extract a test solution with the salts from the surface, and the second is to analyze the solution to determine the concentration of the salt on the surface.

In the case of the saturated filter paper extraction method, cleaned filter papers are wetted with a controlled volume of pure water and the paper is then placed on the surface to extract the soluble salts. The paper is left on the surface for 2 minutes and then it is removed from the surface and placed on the electrode of the conductivity meter. The meter then tests the conductivity for this known area of the filter paper and the known volume of test solution. The result is displayed by the gauge as a value in $\mu \mathrm{g} / \mathrm{cm}^{2}$. Figure 2 shows a schematic view of the measurement process for the saturated filter paper extraction method.

The soluble salt concentration includes the weight of both cation and anion. The reading is therefore derived from a total conductivity measurement. The gauge converts the total conductivity reading over the known area of the sample paper, calibrated as sodium chloride. To convert the reading to a chloride ion value assuming that sodium chloride is the only source of chloride ions, the factor will be 1.65 based on atomic weights.

Therefore a reading of $2.0 \mu \mathrm{g} / \mathrm{cm}^{2}$ total salt level on the instrument would be divided by 1.65 to give $1.2 \mu \mathrm{g} / \mathrm{cm}^{2}$ as chloride.

Using the Saturated Filter Paper Extraction Method

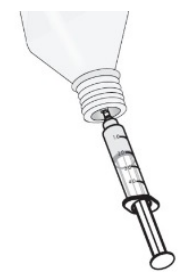

Fig. 1. Fill the syringe with $1.6 \mathrm{ml}$ of high purity water.

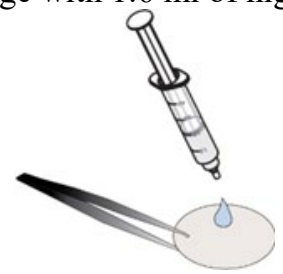

Fig. 2. Eject the $1.6 \mathrm{ml}$ on to a clean unused sample paper, taking care to retain all the water on the paper.

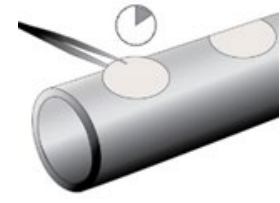

Fig. 3. Place wetted paper on to the area under test, pressing firmly into contours and irregularities to remove any entrapped air.

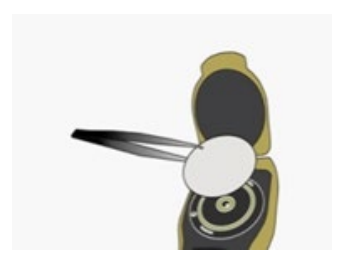

Fig. 4. After 2 minutes, remove the paper from the surface and place it on to the electrodes.

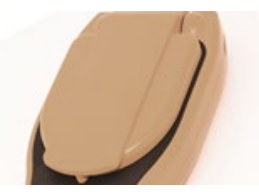

Fig. 5. Close the lid.

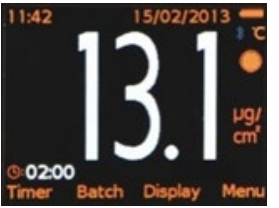

Fig. 6. The reading will be displayed.

In line with several modern electronic coating inspection gauges, the latest design of conductivity meter makes use of microprocessor electronics to enable operational features to be added to make the measurement of surface salt concentration and the management of the resulting data quicker and easier. Microprocessor electronics allow the gauge to be operated from a menu displayed on a large color LCD, with on screen charts and step-by-step guidance for the user in different languages and four large buttons that change their function depending on the area of the menu selected.

The gauge case is hand-held and fully portable for field use and is designed to be dust and water resistant to IP64 equivalence. The gauge uses large control buttons so that the gauge is easy-to-use when wearing gloves. 


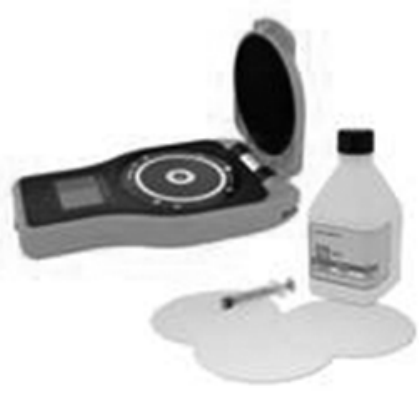

Fig. 7. The main parts of the salt contamination test kit

Having confirmed the cleanliness of the substrate the coating can be applied but the climatic conditions need to be acceptable for applying a coating.

It is now possible to determine the ambient climatic conditions using a rugged electronic gauge that contains all the functionality to make the "paint or don't paint" decision accurately and very quickly in a single gauge. An example of this type of gauges is shown in figure 3 below.

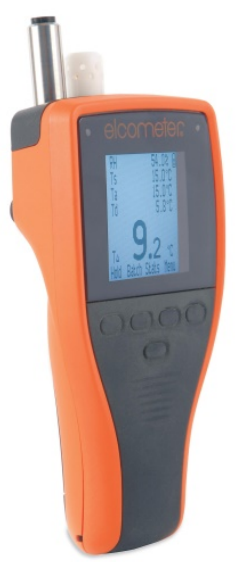

Fig. 8. Digital electronic dewpoint meter

The Digital Electronic Dewpoint Meter is described in BS EN ISO 8502-4 as digital electronic hygrometers based on measurement of capacitance change of polymer films.

\section{The digital electronic dewpoint meter}

Digital humidity gauges have been available for some years but there is now a Dewpoint Meter that is designed specifically to monitor the paint process and that also provides a method for recording and presenting data as proof of the conditions prevailing.

The Digital Electronic Dewpoint Meter measures relative humidity $(\mathrm{RH})$, air temperature $(\mathrm{Ta})$ and surface temperature (Ts). Using the rh and air temperature, the gauge calculates the dewpoint temperature (Td) and then calculates the difference between the dewpoint temperature and the surface temperature $(\mathrm{T} \Delta)$ and assess this difference against the $5^{\circ} \mathrm{F}\left(3^{\circ} \mathrm{C}\right)$ threshold that is used to determine if it is safe to paint.

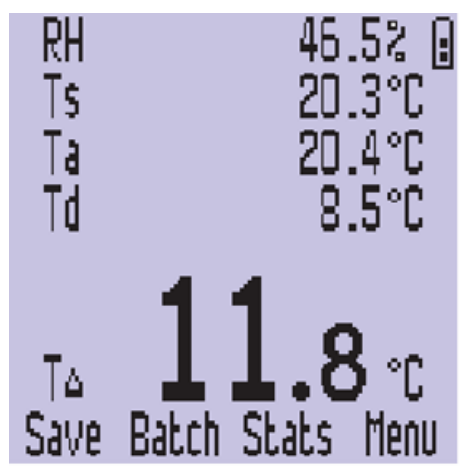

Fig. 9. Electronic dewmeter display showing the RH, Ts, $\mathrm{Ta}, \mathrm{TD}$ and $\mathrm{T} \Delta$ values on screen together

A semiconductor sensor is used to measure the relative humidity so the measurement response time is much quicker than the traditional wet and dry bulb method that can take 20 minutes or more to reach steadystate conditions, at which point the temperatures of the bulbs can be read. This is particularly the case when the hygrometer has been stored in office conditions and then is used outdoors in different conditions of temperature and humidity. Even when an Electronic Dewmeter is taken from a warm office or car to a cold environment, an accurate reading of rh can be obtained in less than 30 seconds.

The air temperature is measured using another semiconductor sensor mounted close to the RH sensor to minimise any differences in conditions. The output from this sensor is displayed as Ta. The surface temperature is measured either using an integrated K-type thermocouple sensor mounted next to the RH and Air Temperature sensors or by using an optional external thermocouple sensor connected via a K-Type connector. This sensor can be also be used for measuring the temperature of materials to be used in the process or for surface temperature monitoring during the process, datalogging. When an external thermocouple sensor is connected the integrated surface temperature sensor is disconnected from the gauge. (See figure 4).

In some instances, test records for climatic condition monitoring require that the wet and dry bulb temperatures are entered into the record sheet so to make this process easier and quicker the Electronic Dewmeter provides estimates of the relevant wet and dry bulb temperatures based on the measurement of $\mathrm{RH}$ and Ta. The estimated values for the wet bulb temperature (Twb) and the dry bulb temperature $(\mathrm{Tdb})$ can be displayed by selecting these parameters from the Display menu in preference to two of the usual set, e.g. Ta and T $\Delta$. Note Ta will be the same value as Tdb.

The digital format of the gauge allows sets of reading to be stored in memory for further analysis, reporting or archiving. Each set of readings consists of an RH value, 
the corresponding air temperature, surface temperature and the calculated Dewpoint Temperature and temperature difference, together with the estimated values for wet bulb and dry bulb temperatures.

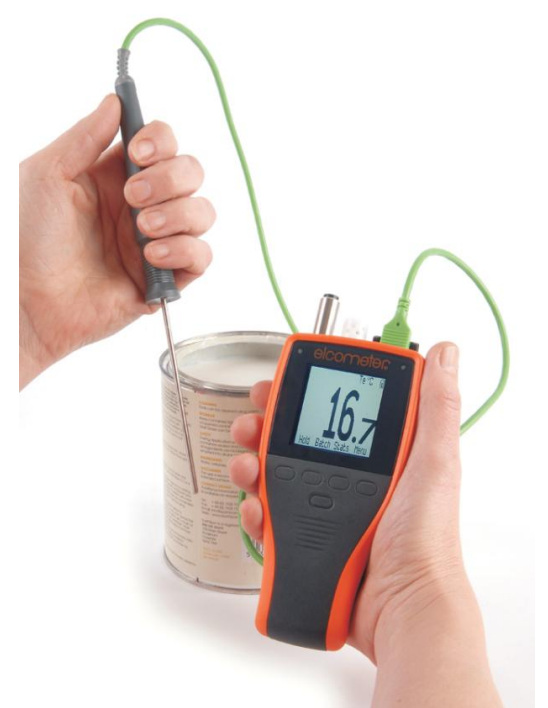

Fig. 10. The electronic dewmeter with an external thermocouple sensor connected.

The memory capacity is a maximum of 25,000 records or sets, in up to 999 different batches. Data can be collected either on command by pressing "HOLD" or using the preset interval data-logging feature. The preset interval can be adjusted from 1 second to 1 hour.

High limits and low limits can be set for each of the climatic parameters; RH, Ta, Ts and Td. High and low limits can also be set for $\mathrm{T} \Delta$ but a default low limit of $3.0{ }^{\circ} \mathrm{C}$ is preset in Celsius units with an equivalent of 5.4 ${ }^{\circ} \mathrm{F}$ in Fahrenheit units. When set and activated by setting the high or low limit on in the "LIMITS" menu, the gauge gives audible and visual warnings when a limit is exceeded.

The display also indicates trends for the values on the screen; with the $\boldsymbol{\Delta}$ symbol indicating an increasing trend fro the value and the $\boldsymbol{\nabla}$ symbol indicating a decreasing trend.

Data can be recorded using two methods; (i) user activated recording of values as and when required or (ii) the data-logging mode, with reading sets recorded automatically at the end of a pre-set interval.

In the data-logging mode the gauge is attached to the steel substrate by three strong magnets mounted in the back of the gauge case. The magnetic external thermocouple is also attached to the substrate

This arrangement is shown in figure 5 below.

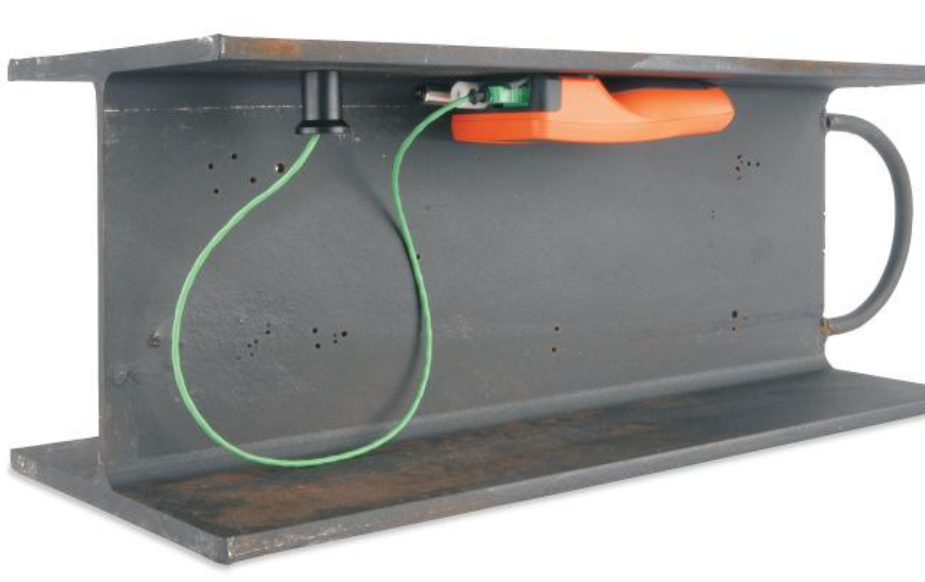

Fig. 11. Dewpoint meter configured for data-logging

The User operates the manual data collection mode via the manual logging batch option. After taking readings the user presses the "HOLD" softkey to lock the measurements on the screen. The options to "Save" or "Escape" (Esc) will be then be displayed and the user can choose to save the data or 'escape' and not store the readings.

Similarly the data-logging option can be chosen when a new batch is created. Adjusting the logging interval can be done on-screen. The default time is 1 minute and can be adjusted to be a shorter or a longer time interval to suit the user/application.

Once data has been collected either in manual or data-logging time interval mode the statistical analysis of the reading sets can be viewed on screen. These statistics include the number of readings taken, the mean, the standard deviation, the coefficient of variation, the highest and lowest readings are displayed with a separate page for each climatic parameter, RH, Ts, Ta, Td, Ts, $\mathrm{Tdb}$ and $\mathrm{Twb}$. These pages can be scrolled through by the user.

\section{Dry Film Thickness}

When measuring protective coatings applied to metal structures, accuracy and speed of data collection are key aspects. The new coating thickness gauge approach to the measurement of coating thickness on a profiled steel surface allows for pre-programmed memories to facilitate the accurate adjustment and setting of the gauge for data collection along with a scanning probe to enhance the speed of operation.

Many electronic coating thickness gauges can store coating thickness readings in batches and these batches can be created using a pre-programmed calibration that simplifies the process of making new batches for a specific substrates profile. Typically there are three adjustment setting that correspond to the three levels of profile as defined in ISO 8503-1.

When using the two-point adjustment method while creating the calibration memory will ensure that the gauge accurately reads the coating thickness over the profile beneath. 
The second recent development is that of the scanning probe for continuous coating thickness measurement. This new design of probe allows for a 'snap on' replaceable end cap to prevent wear of the probe tip as it is dragged over the surface being measured. The wear cap maintains the accuracy of the probe and increases it's working life as even relatively soft coatings will cause wear and damage to any unprotected probe. The scan probes use a patented offset feature in the gauge, this ensures that the thickness of the wear cap is calibrated out of final measurement value of the coating. The gauge will also display a warning message when the cap has has reached the point where the cap should be replaced as it is worn through use.

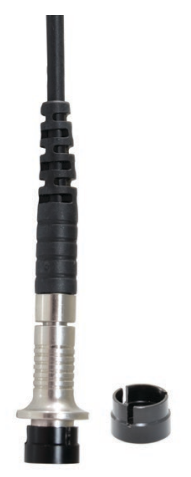

Fig. 12. Example of scanning probe. While scanning the gauge is taking 140 readings per minute.

The gauge firmware has been enhanced to allow the scanning probe to operate in several modes for swift data collection into the memory of the gauge. In the first mode the probe can slide over the surface of the coating and when the probe is lifted the gauge stores all of the readings taken and displays the average coating thickness value, the highest thickness value and the lowest thickness value. Other scans over the surface will continue to add readings to the memory and the gauge will calculate the average, and display the maximum and minimum value for the total number of readings in the batch

In the second mode the gauge can be set to scan over the surface with the gauge collecting all the readings until the probe is lifted off the surface. At this point the gauge will store only the average of those readings along the maximum and minimum reading in that unique scan. Repeated scans will create a second measurement point in the memory that is the average and maximum and minimum reading of the second scan. This continues until the batch is closed.

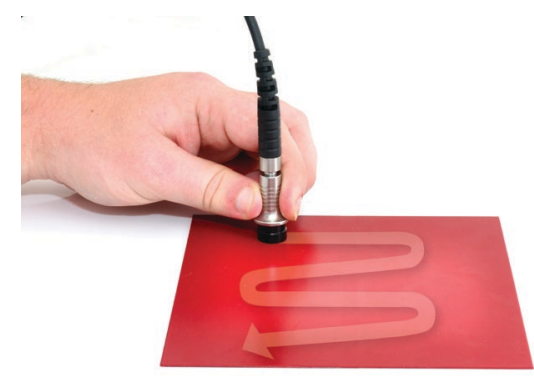

Fig. 13. A scanning probe in use

The firmware also has a Counted Average function and using this with the scanning probe significantly speeds up the taking of the readings set as the counted average.

Additionally the Fixed Batch Size function allows the number of readings required to complete each batch to be defined by the user. The gauge will automatically create a new (linked) batch once the fixed batch has been created.

Job specifications for the determination of coating thickness often describe the number of individual thickness gauge readings to be taken as a spot measurement and the number of spot measurements for a given area. For example, SSPC PA- $2^{i}$ requires three gauge readings per spot and five spot readings in a $10 \mathrm{~m}^{2}$ $\left(\sim 100 \mathrm{ft}^{2}\right)$ area.

With the Scan Probe and the Auto Repeat function, data that is compliant with the SSPC PA-2 method can be collected at a rate that is more than $40 \%$ faster than with the more traditional manual measurement method.

A counted average setting of three readings and a fixed batch size of five spot readings allows the coating thickness reading data, defined by SSPC PA-2, to be recorded for each inspection area and stored in its own batch memory. With the linked batches providing data for the whole structure that is being assessed. This feature also has the additional benefit of allowing each batch to be named to identify the location of the readings so that any corrective action can be limited to the area of concern, rather than being applied to the whole job.

Another feature of the scanning probe is a 'hold' function. This allows the probe to be removed from the surface for up to 1.5 seconds and then replaced on the surface to continue with the same scan; allowing for the probe to be lifted from the surface to clear obstacles such as welded joints and other obstructions.

\section{Adhesion Measurement}

The Type V Adhesion tester is operated by hydraulic pressure 


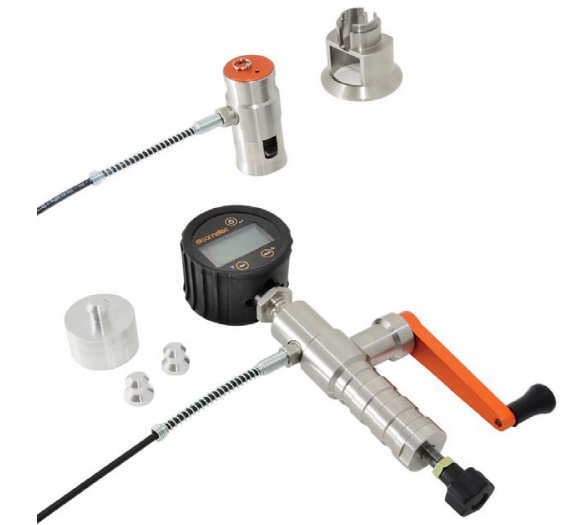

Fig. 14. Manual Type V adhesion tester management software such as ElcoMaster ${ }^{\mathrm{TM}}$ for PC and Apps for iPhone, iPod, iPad and Android mobile devices All pull-off adhesion test standards recognise that it is not only the value of the strength of the coating that needs to be recorded but also the mode of failure is significant and should be recorded/reported. This failure mode is treated as attribute information and can be added to each individual pull test stored in the batch.

First and foremost, a glue failure is not a valid reading unless the force applied to achieve the failure shows a value that is above the value specified for the coating, in which case the coating can be said to have exceeded the specification for the adhesive strength of the coating.

Typically a glue failure with be an invalid test (glue fails below the required strength of the coating) and the test will have to be repeated. Allowance for possible glue failures can be included in the number of dollies applied to an inspection area.

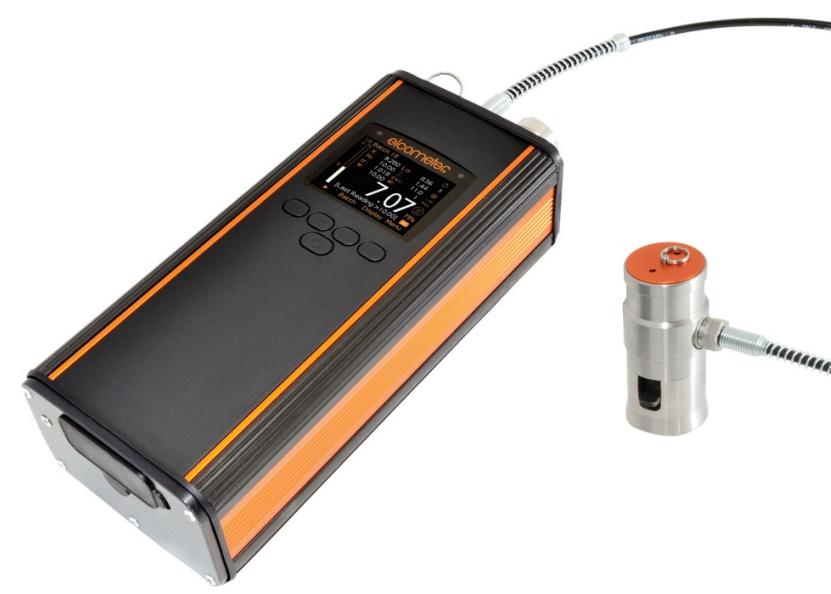

- When the force ap specified value for the coating it is not always necessary to continue until the coating fails, as this will require a repair to the coating and such a test will not provide any additional information. There is a feature in the gauge where the dolly can be pulled to a limit - the limit being the expected/required strength of the coating.

If the dolly is pulled to the set limit and the coating does not fail the gauge will release the force from the dolly leaving the coating in tact and the dolly can be removed using heated tongs to melt the adhesive.

If the fracture strength is less than the specified value the validity of the test is determined by visually inspecting the face of the dolly and determining the surface area and mode of the coating failure.

If no more than $20 \%$ of the area of the face of the dolly shows a coating failure of the adhesive and or cohesive property of the coating or the glue or adhesive failure at the glue/topcoat interface the test is not valid.

For a fracture to be identified as adhesive or cohesive the area of the relevant fracture must be $80 \%$ or more of the dolly face.

The following panel describes how the attribute data is coded and how the Elcometer 510 Automatic Adhesion Tester can be used to manage this information.

\section{Assessing The Results - Failure Attributes}

Many National and International Standards, including ISO 4624 \& ASTM D4541, require the user to record not only the pull-off force but also the nature of the failure. This is done by examining the bottom of the dolly and assessing the failure. In 'Advanced' mode on the Elcometer 510 it is possible to select the 'Attributes' feature (Menu/Setup/Gauge Mode/Advanced) allowing the nature of the fracture to be recorded against each reading and stored within the batch. 


\section{Examining The Dolly}

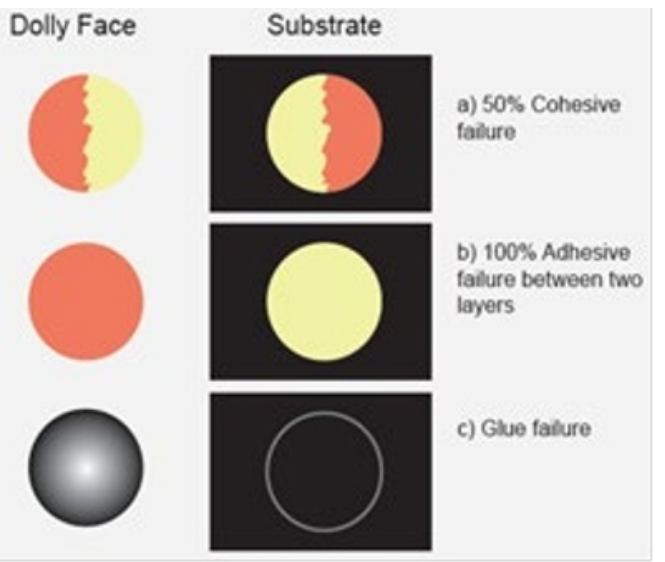

Fig. 16. Dolly face appearance - attributes

a) Cohesive Failure: The coating fails within the body of a coating layer leaving the same coating on the surface and on the dolly face.

b) Adhesive Failure: Failure occurs at the interface between layers (inter-coat) where one pulls away from the other. The "coating" on the dolly face will not be the same as that on the test area.

c) Glue Failure: When no coating is present on the dolly it must be recorded as a failure of the glue. This may be due to incorrect or insufficient mixing of the component parts of the adhesive, incompatibility between the adhesive/coating/dolly/test surfaces.

\begin{tabular}{cccl} 
Cohesive Failure Layers & \multicolumn{2}{c}{ Adhesive Failure Layers } \\
Code & Description & Code & Description \\
A & Substrate & A/B & Substrate \& Layer 1 \\
B & Layer 1 & B/C & Layer 1 \& Layer 2 \\
C & Layer 2 & C/D & Layer 2 \& Layer 3 \\
D & Layer 3 & D/E & Layer 3 \& Layer 4 \\
E & Layer 4 & E/F & Layer 4 \& Layer 5 \\
F & Layer 5 & F/Y & Layer 5 \& Glue \\
Y & Glue & Y/Z & Glue \& Dolly
\end{tabular}

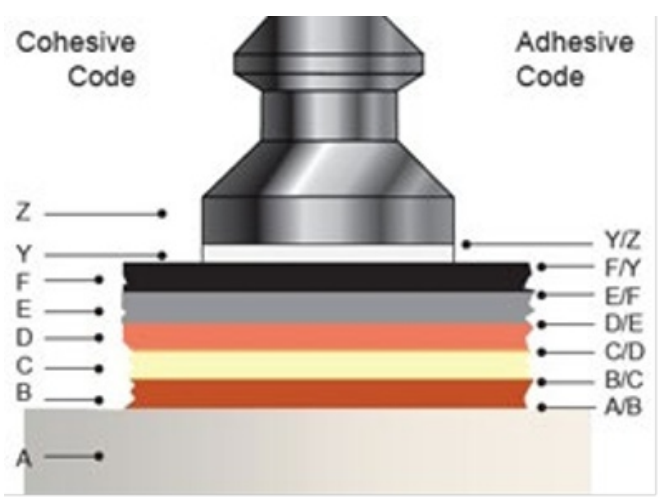

Fig. 17. Coating layers and failure codes

\section{Porosity assessment}

The new design of Pulsed DC High Voltage Holiday Detector allows coatings to be tested using a training signal return cable that lies on the surface of the coating and provides a capacitive signal path if a flaw is located by the high voltage electrode.

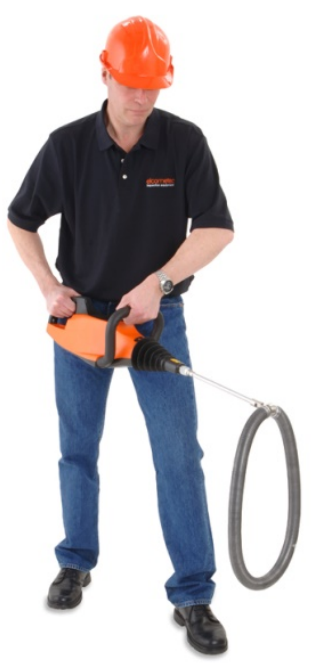

Fig. 18. The pulsed DC high voltage holiday detector showing a rolling spring electrode ready to wrap round a pipe.

The trailing signal return cable is able to operate because the Pulsed DC test voltage is changing, rising from zero volts to the pre-set test voltage, several $\mathrm{kV}, 30$ times per second. This changing voltage means that a capacitor in the circuit will charge and discharge allowing current to flow. This is not the case with the Continuous DC power supply. A training lead on top of the coating will create a capacitor between the conductive substrate and the un-insulated cable conductor with the insulating coating acting and the dielectric in the capacitor.

It is also the case that the energy in the Pulsed DC system is contained within the short duration pulses and therefore the test voltage can be maintained on a slightly conductive coating. The system is monitoring for a significant release of energy through a flaw and can ignore the lower energy released by dirt or moisture on 
the coating. The alarm circuit is set to ignore these stray currents and only react to the significant pulses of energy in the signal return cable.

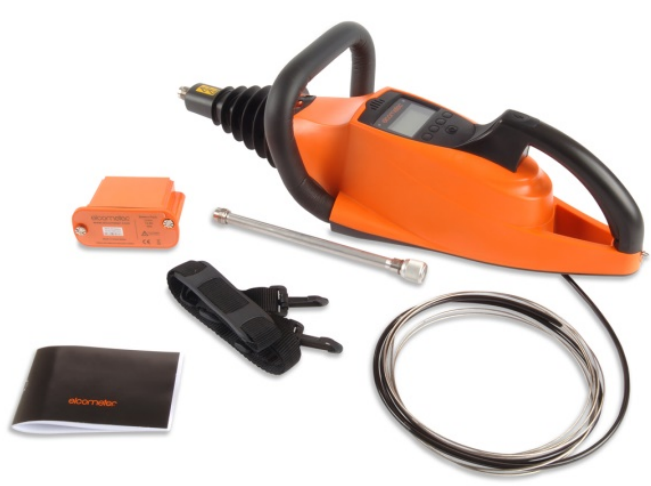

Fig. 19. A pulsed DC system showing the trailing lead, the battery pack, the extension piece, the shoulder strap and the operating instruction

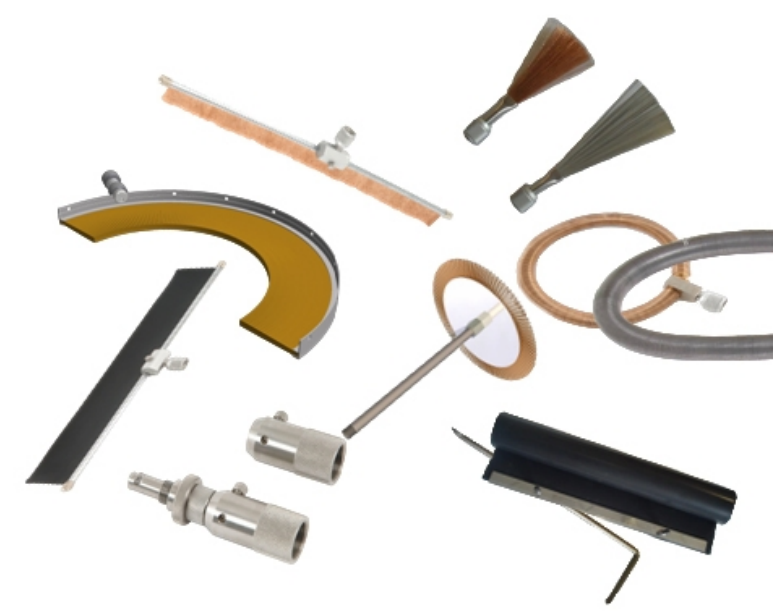

Fig. 20. An array of high voltage test electrodes including band brushes, rolling spring, straight and curved wire brushes, conductive rubber and internal brush types. These can be used with either continuous DC or pulsed DC high voltage porosity detectors.

It should be noted that the capacitive effect that provides the connection through the trailing lead also has an influence on the choice of test electrodes. The capacitive loading of a particular electrode design is affected by the size of the electrode and the thickness of the coating to be tested. In some cases, a large electrode on a thin coating can provide a significant capacitive loading to the pulsed high-voltage power supply making it appear that the electrode is constantly finding flaws. A smaller electrode will normally solve this problem.

Good quality control procedures will provide for the monitoring of the surface preparation processes, the climatic conditions at the time of the application and the process outcomes, such as wet film and dry film thickness, porosity, adhesion and where appropriate appearance, colour, gloss, etc. The issue for the client is the provision of evidence that these monitoring or inspection processes have been carried out correctly and ensure the best quality outcomes consistently.

This is leading to increased demand for detailed reports on the individual stages of the coating process rather than the simple pass/fail statements of the inspector that has prevailed in the past. Often the "evidence" has been a list of hand-written values recorded in the inspector's logbook for the day that the work was carried out.

Some of the coating process inspections can be carried out using digital gauges and therefore inspection areas can be associated with batches of readings and these provide good evidence of the work and the outcome. Other inspection processes are only possible using manual gauges where the Inspector has to note the result. For example, the Bresle Salt Contamination Test according to ISO 8502-6 and ISO 8502-9 produces a result on a gauge without data output capabilities. There are also a group of inspection tasks that rely on the expertise and experience of the Inspector and these are assessed against visual standards or guides, such as the Swedish Rust Standard (ISO 8501-2) or the SSPC VIS 1 guide and reference photographs. The results of these inspections will be a simple pass/fail statement against the specification, for example does the surface preparation meet the Sa $2^{1 / 2}$ rating as defined by ISO 8501-2 or alternatively the commercial blast grade described in SSPC VIS 1.

The problem is therefore, how can good evidence of the inspection results for coating processes be provided without adding considerable cost to the process?

\section{The Data Management Solution}

The answer to the need for a cost effective evidence trail for coating inspection processes is in the planning of the inspection tasks and the recording and presentation of the inspection results. This is commonly called "Paperless QA" and relies on computer software solutions.

There is a new development in the software available for managing and reporting inspection data in the industrial environment. Software has now been developed to operate in either standard mode to facilitate data transfer, data viewing and simple data reports, or in the extended mode, which also provides further analysis with combination of batches of data and inspection templates and customised report creation.

The software has several language sets for the messages, instructions and menus so operation in the international environment is simplified. For example, the software can be set up for Spanish, French or German operatives with similar menus in Spanish, French or German on the digital gauges that link to the software.

The software opens with the data download screen, which allows the selection of a range of inspection gauge types. These include dry film thickness gauges, a coating hardness tester, an appearance gauge (Glossmeter), material thickness gauges (Ultrasonic Gauges), surface profile gauges and a climatic monitoring gauge. 


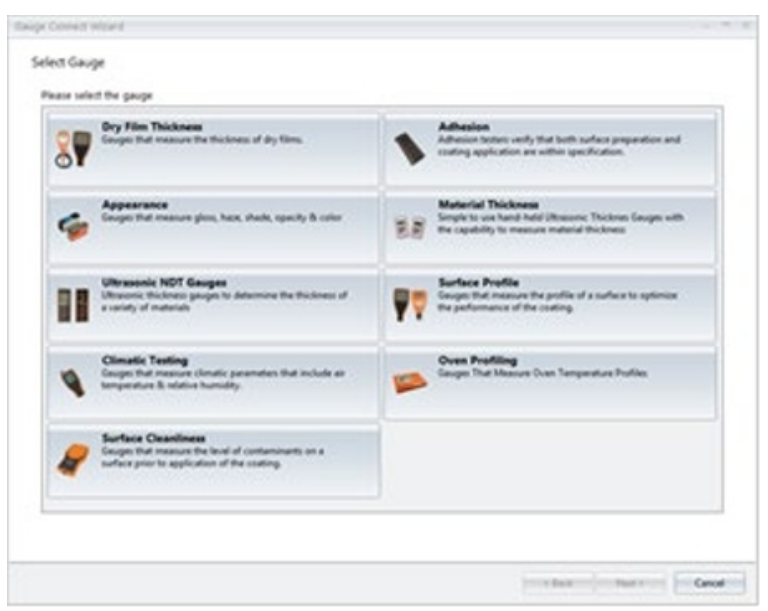

Fig. 21. The gauge selection screen showing the major gauge options

The data transfer can be achieved using Bluetooth ${ }^{\circledR}$ wireless data transmission, USB data cable or the RS 232 serial data cable depending on the capability of the gauge and the computer being used. The data transfer and other software features are supported by a "Wizard" to simplify and speed the process, with on screen instructions for clarity.

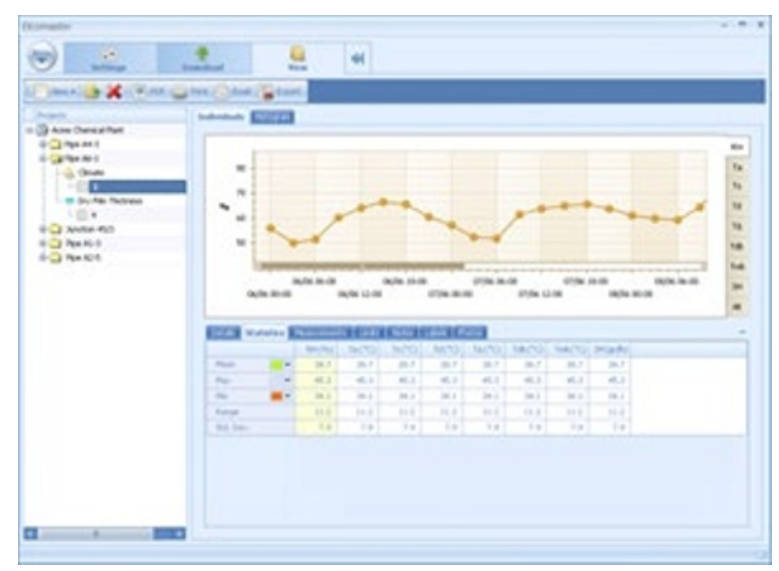

Fig. 22. A single page data report showing a run chart

The software has a top-level folder in the directory, which is called "Project". This can be used to identify the work item for which the inspection data is being collected. In this way data from different gauges and different inspection stages can be linked together to eventually produce a single report on multiple inspection stages. Each project can have three project labels and five batch tags to facilitate grouping of data for further analysis, e.g. all the coating thickness readings on the night shift, etc.

Once data has been transferred from the gauge to the software, the view page offers the capability to see various charts compiled from the batched values, details of the batch (gauge information and statistics), reading limits, notes, labels and photographs.

Data from the different styles of gauges can be combined into a single report so that the surface profile data for a component can be seen alongside the climatic conditions at the time the paint was applied, with the climatic conditions during the cure process and the final film thickness data.

The SSPC Average takes groups of dry film thickness readings, normally three, and averages them in to a single reading, known as a spot reading. These averages readings are then grouped and averaged. This analysis is not valid for climatic reading sets.

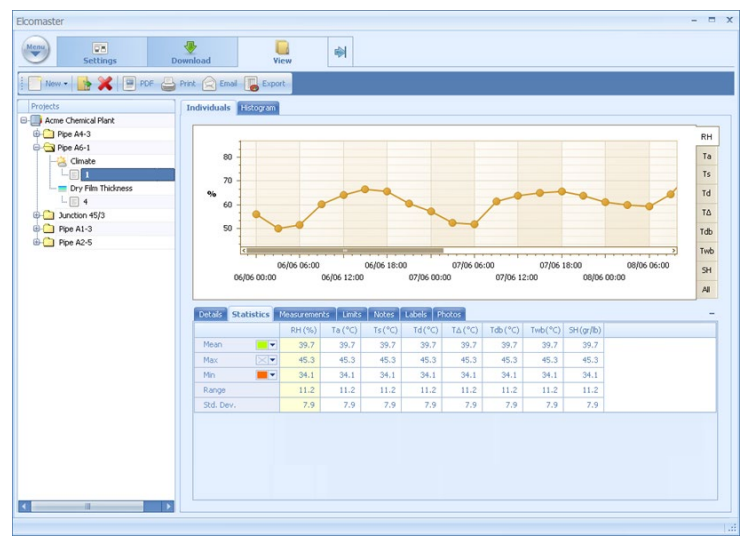

Fig. 23. An example of the statistics page for climatic data

Batches can be combined to form a larger batch for further analysis. For example, if a structure has been divided up into significant areas for inspection and each area given an individual batch number, a group of batches can be combined to determine the overall average value or the highest and lowest values, etc.

It is also possible to create templates so that with structured data collection the relevant readings can be shown against their location on the component for more detailed analysis. The positioning of the reading sequence can be set so that the operator can take batches of data with reading 1 in the same position for each component or part of the structure. This could be peakto-valley profile readings on a plate or coating thickness readings on a beam.

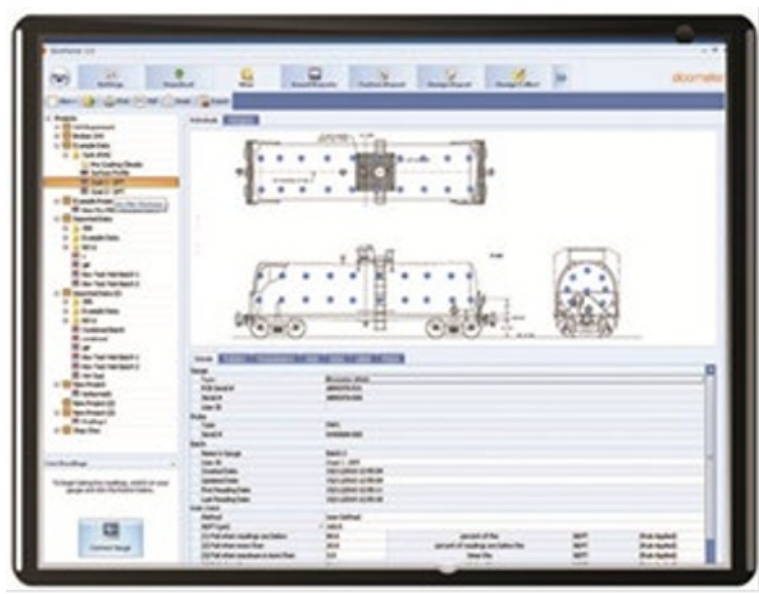

Fig. 24. A single page coating thickness report form with inspection template 
Information can be printed, e-mailed or compiled as a .pdf file for reporting and it is possible to export the readings that have been downloaded to the software either directly into a Microsoft ${ }^{\circledR}$ Excel spreadsheet or into other spreadsheet formats using CSV (comaseparated values). The print command uses the computer's default printer. The e-mail command opens an e-mail message and appends the data report and the PDF command converts the report to the Portable Document Format (PDF) that allows two-dimensional documents to be represented in a manner, which is independent of the application software, hardware and operating system.

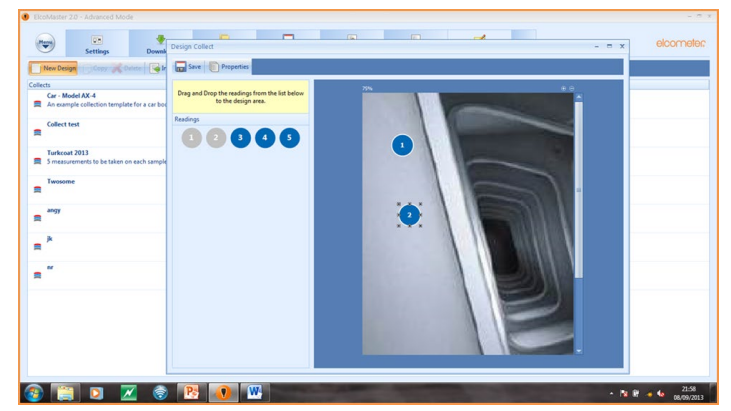

Fig. 25. The template set-up showing the positioning of the reading sequence

A recent innovation is the addition of "Cloud" computing to make it possible to share data files and reports by transferring them to the Internet via a cloud drive. The Cloud allows data to be transferred, stored and accessed through an Internet provider's hardware, without the need for a local computer. There are a number of Cloud providers such as Dropbox, Amazon Cloud Drive, Google Drive, SkyDrive, etc. and these services can be accessed via the data management software using both personal computers and mobile Internet capable devices.

Imagine an Inspector working at a remote site with no access to a computer network, or a number of inspectors working at different places on a large site or even on different sites, all needing to communicate data to a central point quickly. It would even be practical for several inspectors at several sites in different countries with a client in yet another country to communicate quickly and simply using the cloud drive as a commonly accessed database resource.

The inspection measurements are taken on the structure and stored in the memory of the gauge. These readings are then transferred from the gauge to any Smart phone or tablet using Android via the Bluetooth ${ }^{\mathrm{TM}}$ communications and using the Android-based ElcoMaster ${ }^{\mathrm{TM}}$ Mobile software. This data can then be emailed to the Cloud. The specific Cloud drive is then accessed by any authorised device in any country on any continent to share the information, quickly, accurately and at a low cost.

Using these techniques inspection information to be shared instantly and therefore reporting time and costs are greatly reduced. Decision-making, based on good quality information, can be timely and accurate, particularly when re-working is indicated. At the end of a coating process, approval for the next stage can be quickly given, even when the client is at the other side of the world.

Bluetooth $^{\mathrm{TM}}$ has been preferred to $\mathrm{Wi}-\mathrm{Fi}$ for the ElcoMaster 2.0 application because it does not suffer from the requirement to dedicate the communication link to a single task such as data transfer from a gauge to a suitable device. For example, if Wi-Fi is used to transfer data to a Smartphone with the intention of sending it to the Internet, all other Wi-Fi connections on the Smartphone, such as to a Wi-Fi hub or to a Wi-Fi headset or to a Wi-Fi hands-free system, must be completely disconnected until the transfer is complete. The link to the Internet from the Smartphone then has to be re-established before the Cloud can be accessed. Bluetooth can be used for multiple communications simultaneously and is password protected for security. It is also the case that the current consumption of a Wi-Fi connection in a battery powered hand held gauge is very significantly higher than that of a Bluetooth connection.

The Cloud database offers a significant expansion in the way coating inspection data is transferred and viewed utilising equipment such as a Smartphone, which is already part of the travelling Coating Inspector's normal kit.

The Report Designer software feature is also available in the Extended Mode of the software and allows the User to create a customised report format specific to the client or the work in hand. The report can reference a single batch of data or multiple batches. The software uses a wizard to aid the setting up of a Report Design and each report has a unique name and description. The wizard will then request what type of data is to be included and how many batch of each type. Finally, the design page is displayed.

The report designer comprises of a design area, a list of pages, and a list of report items from which the design area is populated. The report item list contains a basic set of report items, such as a text box, an image, etc. These are dragged from the item list onto the design area, once dragged these can then be positioned and resized to suit.

In addition to the general report items, are items specific to the batch types, previously specified in the Wizard. Therefore, if the user specification for this report was for a single coating thickness batch, only the coating thickness readings, statistics \& charts are dragged to the design area.

It is also possible for the user to click and drag the project, folder and batch labels onto the design area, alongside the batch \& general report items.

Any relevant information modification appears above the selected item in the form of a toolbar. So with regards to text, the font \& style can be changed, whereas with an image, the method used to re-size the image can be changed. Pages can be added and deleted from the report via the toolbar. 